\title{
PRETZELS INTEGRAIS ADICIONADOS DE INULINA: AVALIAÇÃO FÍSICO- QUÍMICA E SENSORIAL ENTRE CRIANÇAS
}

\author{
Thaís SOLAGNA ${ }^{1}$ \\ Bruna Magusso RODRIGUES ${ }^{2}$ \\ Nathália Medina dos SANTOS ${ }^{2}$ \\ Camila Jordão CANDIDO ${ }^{3}$ \\ Elisvânia Freitas dos SANTOS ${ }^{4}$ \\ Daiana NOVELLO 5
}

${ }^{1}$ Graduanda em Nutrição, Universidade Estadual do Centro-Oeste (UNICENTRO).
${ }^{2}$ Graduanda em Nutrição, Universidade Federal de Mato Grosso do Sul (UFMS).
${ }^{3}$ Farmacêutica, Mestra, Técnica em Alimentos e Laticínios (UFMS).
${ }^{4}$ Professora, Doutora, Departamento de Nutrição (UFMS).
${ }^{5}$ Professora, Doutora, Departamento de Nutrição (UNICENTRO). *E-mail: nutridai @ gmail.com.

Recebido em: 17/06/2015 - Aprovado em: 09/01/2016 - Disponibilizado em: 30/07/2016

RESUMO: O presente estudo objetivou verificar a aceitabilidade sensorial de pretzels integrais adicionados de inulina e determinar a composição físico-química da formulação padrão e daquela contendo maior teor de inulina e com aceitação sensorial maior que a padrão. Foram desenvolvidas 5 formulações de pretzels: F1 (padrão - 0\%) e as demais adicionadas de $1 \%(\mathrm{~F} 2), 2 \%(\mathrm{~F} 3), 3 \%$ (F4) e 4\% (F5) de inulina. Participaram da avaliação sensorial 132 provadores não treinados, de ambos os gêneros, com idade entre 7 a 10 anos. As formulações F3, F4 e F5 tiveram maiores notas de aceitabilidade $(\mathrm{p}<0,05)$ que F1 e F2 para os atributos de aparência, aroma, sabor, textura e cor. Já para a aceitação global, F4 e F5 mostraram maior aceitabilidade que F2 e F3, sendo que F1 apresentou a menor nota pelos provadores. No teste de intenção de compra observou-se que F4 e F5 tiveram maiores notas que F1 e F2, sendo que, de forma similar, F1 também foi a menos favorável neste quesito. Não houve diferença significativa ( $>00,05)$ entre F1 e F5 em relação aos teores de cinzas, proteínas e lipídios. Entretanto, maiores teores de umidade e fibra alimentar e menores de carboidratos e calorias foram verificados em F5 quando comparada a F1. Assim, a elaboração dos produtos permitiu comprovar que um nível de adição de até $4 \%$ de inulina em pretzels foi bem aceito pelos provadores infantis, obtendo-se aceitação sensorial semelhante ao produto padrão e com boas expectativas de comercialização.

Palavras-chave: Farinha de linhaça. Alimentos funcionais. Cereais.

\section{Whole pretzels added inulin: physico-chemical and sensory evaluation among children}

\begin{abstract}
This study aimed to verify the sensory acceptability of whole pretzels added inulin and determine the physico-chemical composition of standard formulation and that containing more inulin content and sensory acceptance higher than standard. Five pretzels formulations have been developed: F1 (standard- 0\%) and the others added 1\% (F2), 2\% (F3), 3\% (F4) and 4\% (F5) inulin. Participated in the sensory evaluation 132 untrained, of both genders, aged between 7 10 years. The formulations F3, F4 and F5 had greater acceptance of notes $(p<0.05)$ than F1 and F2 to the attributes of appearance, aroma, taste, texture and color. As for the global acceptance, F4 and F5 showed greater acceptance that F2 and F3, and F1 had the lowest score by the judges. In purchase intention test it was noted that F4 and F5 had higher scores than F1 and F2, and, similarly, F1 was also less favorable in this regard. There was no significant difference ( $p>0.05$ ) between F1 and F5 in relation to ash, proteins and lipids content. However, higher moisture content and dietary fiber and lower in carbohydrates and calories were checked in F5 when compared to F1. Thus, the development of products able to prove that a level of addition of up to $4 \%$ inulin in whole pretzels was well accepted by children tasters, resulting in sensory acceptance similar to the standard product with good marketing expectations.
\end{abstract}

Keywords: Flaxseed meal. Functional foods. Cereals.

212

Revista da Universidade Vale do Rio Verde, Três Corações, v. 14, n. 1, p. 212-226, jan./jul. 2016 


\section{INTRODUÇÃO}

A fase escolar se caracteriza por crianças com idade entre 7 a 10 anos (AQUINO et al., 2012). Neste público a saúde pode ser afetada pelas práticas de vida urbana, incidindo em mudanças alimentares, o que aumenta o risco de sobrepeso e obesidade (PONTES AIRES et al., 2011). Também, há um grande consumo de produtos industrializados, o que pode desencadear processos alérgicos e outros distúrbios nutricionais durante na infância. Entretanto, sabe-se que, atualmente, o consumo de fibras por crianças é bem inferior ( $5 \mathrm{~g} / \mathrm{dia})$ ao recomendado (MELLO et al., 2010). Segundo o Institute of Medicine (DRI, 2002), para a população infantil, indica-se a ingestão diária de fibras de $26 \mathrm{~g} /$ dia para meninas e 31 g/ dia para meninos. Neste contexto, o desenvolvimento de novos produtos que apresentem melhora no seu perfil nutricional apresenta grande interesse científico e industrial.

Para satisfazer o novo perfil alimentar dos consumidores, a indústria alimentícia tem realizado alguns avanços com a adição de ingredientes funcionais (PINTO; PAIVA, 2010; SILVA et al., 2011). Os alimentos funcionais são conceituados como aqueles que promovem benefícios ao organismo, por meio de seus componentes ativos, e apresentam boa aceitação por consumidores de diversas faixas etárias. Além disso, alguns alimentos funcionais podem colaborar para a redução do risco de doenças cardiovasculares, diabetes mellitus, patologias do trato gastrointestinal e alergias (CENCIC; CHINGWARU, 2010; RUBILAR et al., 2010).

A linhaça (Linum usitatissimum $\mathrm{L}$ ) é classificada como um cereal integral. É muito estudada por seu elevado valor nutricional e benefícios comprovados para a saúde, sendo considerada um alimento funcional. $\mathrm{O}$ consumo regular de linhaça pode colaborar para a redução do risco de doenças crônicas como a diabetes mellitus e hipertensão arterial sistêmica. Também, possui ação antinflamatória, o que pode diminuir os níveis de colesterol e triglicerídeos sanguíneos (SIMBALISTA et al., 2012; PAULA et al., 2013). A linhaça é composta principalmente por proteínas (14,1 a 21\%), fibras dietéticas (28 a $33,5 \%)$, carboidratos (6\%) e lipídios (32,3 a $41 \%$ ), destacando-se os ácidos graxos $\alpha$ linolênico (n-3) (18\%). Estes ácidos graxos são fundamentais para o adequado funcionamento do sistema digestivo, podendo colaborar para a manutenção dos níveis de glicose e prevenir a constipação intestinal (CAZARIN et al., 2014).

A inulina é outro alimento funcional em evidência. Possui origem vegetal e conceitua-se como um frutano, sendo extraída 
industrialmente, principalmente das raízes da chicória (DALONSO et al., 2009). Classifica-se como uma fibra solúvel, apresentando elevada característica higroscópica (SAAD et al., 2013). $\mathrm{Na}$ indústria de alimentos é bastante utilizada como um substituto de gordura e açúcar, o que se deve ao seu baixo teor calórico $(1,5 \mathrm{kcal} / \mathrm{g})$, elevado teor de fibra alimentar (97\%) $\left(\mathrm{BENEO}^{\circledR} \mathrm{HP}, 2015\right)$ e, geralmente, por não alterar o sabor ou aparência dos produtos (BRASIL et al., 2011). Também, pesquisas que avaliaram a adição de linhaça e/ ou inulina em produtos como biscoitos, iogurtes e bolos demonstraram boa aceitabilidade sensorial (MORAES et al., 2010; MASSODI; KHALID BASHIR, 2012; GANORKAR; JAIN, 2013), o que pode aumentar sua comercialização.

O pão é classificado como um alimento básico e, assim, está no topo dos produtos mais consumidos mundialmente. No Brasil apresenta uma ingestão per capita de $60 \mathrm{~kg} /$ ano (BRASIL et al., 2011), faturamento de R \$ 3,2 bilhões de reais em 2011 (CUNHA, 2014) e elevadas perspectivas de crescimento. Os pães são produtos aceitos por todas as faixas etárias, sendo incluídos em diversas refeições, fato que está diretamente relacionado a algumas de suas características positivas como aparência, aroma, sabor, rendimento e disponibilidade (BRASIL et al., 2011). O pretzel é um pão tradicional alemão, geralmente com formato de nó, assado e salgado. Sua formulação difere do pão comum por apresentar formato, volume e tempo de cocção menor, com destaque para sua leve crocância (OLIVEIRA et al., 2011). Assim, por apresentarem fácil elaboração, propriedades tecnológicas adequadas e grande aceitabilidade, os pães tem se destacado com elevadas possibilidades de melhoramentos e adição de ingredientes funcionais (GANORKAR; JAIN, 2013; IRANSHAHIL et al., 2014).

O desenvolvimento de novos produtos com adição de ingredientes diferenciados requer o uso de ferramentas científicas que avaliem suas características, o que pode otimizar suas possíveis alterações tecnológicas, físico-químicas e sensoriais. No caso da análise sensorial, um resultado positivo de sua aceitabilidade demonstra a satisfação do consumidor com o novo alimento, favorecendo sua possível comercialização (SILVA et al., 2010). Já a análise da composição físicoquímica, é imprescindível para assegurar que o produto atenda aos padrões de qualidade especificados por órgãos nacionais e internacionais, como, por exemplo, conter o rótulo nutricional na embalagem (GOMES; OLIVEIRA, 2011).

O objetivo da pesquisa foi verificar a aceitabilidade sensorial de pretzels integrais com adição de inulina entre crianças, e avaliar a composição físico-química do produto padrão e 
daquele com maior teor de inulina e aceitação superior ao produto padrão.

\section{MATERIAL E MÉTODOS}

\section{Aquisição da matéria-prima}

Os produtos foram adquiridos em supermercados do município de Guarapuava, PR e a inulina foi doada por empresas nacionais parceiras.

\section{Formulação dos produtos}

Foram elaboradas 5 formulações de pretzel: padrão F1 (0\%), F2 (1\%), F3 (2\%), F4 (3\%) e F5 (4\%) de inulina. Estes níveis de adição foram definidos através de testes sensoriais preliminares realizados com o produto. Além das porcentagens de inulina, os ingredientes utilizados nas formulações foram: água $(16,53 \%)$, leite $(16,53 \%)$, farinha de trigo (16\%), ovos $(8,26 \%)$, margarina $(4,96 \%)$, açúcar refinado (F1: 4\%, F2: 3\%, F3: 2\%, F4: 1\% e F5: 0\%), fermento biológico (2,48\%), farinha de linhaça (FL) (2\%), sal $(0,33 \%)$ e farinha de trigo para untar $(28,92 \%)$.

As formulações foram preparadas, individualmente, no Laboratório de Técnica Dietética do Departamento de Nutrição da UNICENTRO.
Inicialmente, foram misturadas em um refratário a inulina, farinha de trigo, água, FL, sal, leite, fermento, açúcar, margarina e os ovos. A massa foi sovada manualmente por 10 minutos, permanecendo em descanso por 50 minutos para fermentação. Em seguida, em uma superfície enfarinhada e lisa, a massa foi cortada em retângulos de $2 \mathrm{~cm}$ de espessura e, depois em tiras de $8 \mathrm{~cm}$ de largura, sendo enrolada para formação dos pretzels. As formulações foram, então, colocadas em assadeiras antiaderentes e coccionadas em fogão convencional (Daltas ${ }^{\circledR}$, Brasil) $\left(230^{\circ} \mathrm{C}\right)$, pré-aquecido, por 12 minutos.

\section{Análise sensorial}

Participaram da pesquisa 132 provadores não treinados, sendo crianças devidamente matriculadas em uma Escola Municipal de Guarapuava, PR, de ambos os gêneros, com idade entre 7 a 10 anos.

Os produtos foram submetidos à análise sensorial em uma sala da escola. Cada prova foi realizada em cabines individuais, tipo urna, na qual o provador foi orientado pelas pesquisadoras para o preenchimento das respostas. Foram avaliados os atributos de aparência, aroma, sabor, textura e cor, através de uma escala hedônica facial estruturada mista de 7 pontos variando de 1 ("Super ruim") a 7 ("Super bom”), adaptada de Resurreccion 
(1998). Foram aplicadas também questões de aceitação global e intenção de compra analisadas através de uma escala estruturada de 5 pontos (1 "desgostei muito"/ "não compraria" a 5 "gostei muito"/ "compraria com certeza"), (MINIM, 2010).

Os julgadores receberam uma porção de cada amostra (aproximadamente $15 \mathrm{~g}$ ), em pratos plásticos descartáveis brancos, codificados com números de três dígitos, de forma casualizada e balanceada, acompanhados de um copo de água para realização do branco. As formulações foram oferecidas aos julgadores de forma monádica sequencial.

O teste de comparação múltipla foi aplicado para comparar as amostras elaboradas na pesquisa com um pretzel comercializado no mercado (referência). Cada julgador identificou se as formulações apresentavam sabor melhor, igual ou pior ao produto de referência em uma escala hedônica estruturada de 5 pontos variando de nota 1 ("muito pior que o primeiro"), a nota 5 ("muito melhor que o primeiro"), adaptada de Dutcosky (2011).

\section{Índice de aceitabilidade (IA)}

O cálculo do IA das formulações foi realizado segundo a fórmula: $I A(\%)=A x$ 100/B (onde: $A=$ nota média obtida para o produto e $B=$ nota máxima dada ao produto) (MONTEIRO, 1984).

\section{Análise físico-química}

As análises físico-químicas foram realizadas no Laboratório de Físico-Química da Unidade de Tecnologia de Alimentos e Saúde Pública (UTASP) da UFMS. As seguintes determinações foram realizadas em triplicata na formulação padrão e naquela com maior nível de adição de inulina e com aceitação sensorial superior a padrão:

Umidade: Foi determinada em estufa a $105{ }^{\circ} \mathrm{C}$ até o peso constante, segundo AOAC (2011); Cinzas: Foram analisadas em mufla $\left(550{ }^{\circ} \mathrm{C}\right)$, conforme AOAC (2011); Lipídios totais: Utilizou-se o método de extração a quente (Soxhlet) (BRASIL, 2005); Proteínas: Foram avaliadas através do teor de nitrogênio total da amostra, pelo método Kjeldahl, determinado ao nível semimicro (AOAC, 2011). Utilizou-se o fator de conversão de nitrogênio para proteína de 6,25; Fibra Alimentar: Foi realizado o cálculo teórico das formulações (TACO, 2011; BENEO $^{\circledR}, 2015$ ); Carboidratos: A determinação de carboidratos dos produtos foi realizada através de cálculo teórico (por diferença) nos resultados das triplicatas, conforme a fórmula: \% Carboidratos $=100-(\%$ umidade $+\%$ proteína $+\%$ lipídios $+\%$ cinzas $+\%$ fibra alimentar); Valor calórico total (kcal): Foi calculado utilizando os seguintes valores: lipídios $(8,37 \mathrm{kcal} / \mathrm{g})$, proteína $(3,87 \mathrm{kcal} / \mathrm{g})$, 
carboidratos $(4,11 \mathrm{kcal} / \mathrm{g})$ (MERRILL; WATT, 1973) e inulina: $1,5 \mathrm{kcal} / \mathrm{g}$ (carboidratos) $\left(\mathrm{BENEO}^{\circledR} \mathrm{HP}, 2015\right)$.

\section{Determinação do Valor Diário de Referência} (VD)

O VD foi calculado em relação a $50 \mathrm{~g}$ da amostra (5 unidades pequenas), com base nos valores preconizados para crianças de 7 a 10 anos (DRI, 2005). Os nutrientes foram avaliados pelo cálculo médio dos provadores, resultando em: 2.080,93 kcal/ dia, 303,70 g/ dia de carboidratos, 78,49 g/ dia de proteínas, $84 \mathrm{~g} /$ dia de lipídios e 15,09 g/ dia de fibra alimentar.

\section{Questões éticas}

Este trabalho foi aprovado pelo Comitê de Ética em Pesquisa da UNICENTRO, parecer número $\mathrm{n}^{\mathrm{o}}$ 608.950/2014. Entretanto, como critérios de exclusão foram considerados os seguintes fatores: possuir alergia a algum ingrediente utilizado na elaboração dos produtos ou não entregar o Termo de Consentimento Livre e Esclarecido (TCLE) assinado pelos responsáveis.

\section{Análise Estatística}

Os dados foram analisados com auxílio do software Statgraphics Plus ${ }^{\circledR}$, versão 5.1, através da análise de variância (ANOVA). A comparação de médias foi realizada pelo teste de médias de Tukey, t de student e Dunnett, avaliados com nível de 5\% de significância.

\section{RESULTADOS E DISCUSSÃO}

\section{Análise Sensorial}

$\mathrm{Na}$ Tabela 1 pode-se verificar $\mathrm{o}$ resultado da avaliação sensorial dos pretzels padrão e acrescidos de inulina.

Tabela 1 - Médias do índice de aceitabilidade (IA) e dos testes sensoriais afetivos de aceitação e intenção de compra, realizados para as formulações de pretzel integral adicionadas de inulina

\begin{tabular}{|c|c|c|c|c|c|}
\hline $\begin{array}{l}\text { Formulações/ } \\
\text { Atributos }\end{array}$ & $\begin{array}{c}\text { F1 } \\
\text { Média } \pm \text { EPM }\end{array}$ & $\begin{array}{c}\text { F2 } \\
\text { Média } \pm \text { EPM }\end{array}$ & $\begin{array}{c}\text { F3 } \\
\text { Média } \pm \text { EPM }\end{array}$ & $\begin{array}{c}\text { F4 } \\
\text { Média } \pm \text { EPM }\end{array}$ & $\begin{array}{c}\text { F5 } \\
\text { Média } \pm E P M\end{array}$ \\
\hline Aparência & $5,55 \pm 0,09^{b}$ & $5,42 \pm 0,08^{b}$ & $6,12 \pm 0,09^{\mathrm{a}}$ & $6,30 \pm 0,09^{\mathrm{a}}$ & $6,22 \pm 0,10^{\mathrm{a}}$ \\
\hline IA (\%) & 79,28 & 77,42 & 87,42 & 90,00 & 88,85 \\
\hline Aroma & $5,55 \pm 0,09^{b}$ & $5,42 \pm 0,08^{b}$ & $6,12 \pm 0,10^{a}$ & $6,30 \pm 0,09^{a}$ & $6,22 \pm 0,10^{\mathrm{a}}$ \\
\hline $\mathrm{IA}(\%)$ & 79,28 & 77,42 & 87,42 & 90,00 & 88,85 \\
\hline Sabor & $5,56 \pm 0,09^{b}$ & $5,40 \pm 0,08^{\mathrm{b}}$ & $6,12 \pm 0,09^{a}$ & $6,31 \pm 0,09^{a}$ & $6,22 \pm 0,10^{\mathrm{a}}$ \\
\hline IA (\%) & 79,42 & 77,14 & 87,42 & 90,14 & 88,85 \\
\hline Textura & $5,58 \pm 0,10^{\mathrm{b}}$ & $5,64 \pm 0,08^{b}$ & $6,17 \pm 0,09^{\mathrm{a}}$ & $6,30 \pm 0,09^{a}$ & $6,39 \pm 0,10^{\mathrm{a}}$ \\
\hline IA (\%) & 79,71 & 80,57 & 88,14 & 90,00 & 91,28 \\
\hline Cor & $5,55 \pm 0,09^{\mathrm{b}}$ & $5,67 \pm 0,10^{b}$ & $6,12 \pm 0,09^{a}$ & $6,24 \pm 0,08^{a}$ & $6,39 \pm 0,09^{\mathrm{a}}$ \\
\hline
\end{tabular}




$\begin{array}{lccccc}\text { IA (\%) } & 79,28 & 81,00 & 87,42 & 89,14 & 91,29 \\ \text { Aceitação global } & 2,83 \pm 0,14^{\mathrm{c}} & 3,41 \pm 0,14^{\mathrm{b}} & 3,48 \pm 0,12^{\mathrm{b}} & 4,37 \pm 0,10^{\mathrm{a}} & 4,26 \pm 0,10^{\mathrm{a}} \\ \text { IA (\%) } & 56,60 & 68,20 & 69,60 & 87,40 & 85,20 \\ \text { Intenção de compra } & 2,63 \pm 0,09^{\mathrm{d}} & 4,22 \pm 0,10^{\mathrm{c}} & 3,91 \pm 0,13^{\mathrm{bc}} & 4,58 \pm 0,09^{\mathrm{ab}} & 4,64 \pm 0,07^{\mathrm{a}} \\ \text { IA (\%) } & 52,60 & 84,40 & 78,20 & 91,60 & 92,80\end{array}$

*Letras diferentes na linha indicam diferença significativa pelo teste de Tukey (p<0,05); EPM: erro padrão da média; F1: padrão (0\%), F2: 1\%, F3: 2\%, F4: $3 \%$ e $\mathrm{F} 5: 4 \%$ de inulina.

Maiores notas foram verificadas para as formulações F3, F4 e F5 $(\mathrm{p}<0,05)$ nos atributos aparência, aroma, sabor, textura e cor. As amostras F1 e F2 não apresentaram diferença significativa entre si. Resultados similares, entre provadores adultos, foram observados por Brasil et al. (2011) que avaliaram pães com adição de inulina (0, 6 e 10\%). Segundo Kaur e Gupta (2002), a maior aceitação dos produtos contendo inulina pode ser explicada por sua composição química, a qual é formada por uma longa cadeia de moléculas de frutose e uma de glicose terminal (FLAMM et al., 2001). Estas substâncias quando dispersas em água ou leite formam microcristais, interagindo entre si, dando origem a uma textura cremosa no produto, fato que colabora para melhorar as características sensoriais.

Para a avaliação de aceitação global, maiores notas foram observadas para F4 e F5, seguidas de F3 e F2. A formulação F1 apresentou a menor aceitação $(\mathrm{p}<0,05)$. Na avaliação da intenção de compra, F5 obteve maior aceitabilidade que F3, F2 e F1. Já, a amostra F4 teve maiores notas que F1 e F2, enquanto F3 e F2 mostraram maiores notas que F1. Resultados similares foram observados por Souza-Borges et al. (2013),

avaliando a aceitação de bolos de laranja e pães adicionados de inulina e oligofrutose. Conforme Kip et al. (2006), mesmo presente em baixas concentrações, a inulina pode influenciar em algumas propriedades, melhorando a aeração e a viscosidade do produto. Esse efeito, provavelmente, colaborou para a maior aceitação e intenção de compra das formulações.

Durante a elaboração dos pretzles foi observado que as formulações com maiores teores de inulina tinham maior viscosidade. Fato que ocorre, também, devido ao elevado teor de fibras presentes neste ingrediente $(97 \%) \quad\left(\mathrm{BENEO}^{\circledR} \mathrm{HP}, 2015\right)$. As fibras apresentam elevada capacidade de absorção de água, formando géis e/ ou agindo como emulsionante, o que colabora para aumentar a viscosidade dos produtos (ELLEUCH et al., 2011). Também, segundo Lipilina e Ganji (2009), a alta absorção de umidade das fibras tem um efeito positivo sobre a textura 
dos produtos alimentícios, principalmente, melhorando a elasticidade e maciez durante seu manuseio e cocção.

Quanto à questão da cor, visualmente, os pretzles contendo maiores teores de inulina apresentaram uma coloração mais escura durante a cocção. Resultados similares foram relatados por Mandala et al. (2009), que avaliaram bolos adicionados de inulina $(5 \%)$ e pães contendo frutooligossacarídeos $(8 \%)$.

Todas as formulações de pretzel apresentaram IA acima de $70 \%$, para a aparência, aroma, sabor, textura e cor o qual classifica o produto com boa aceitação sensorial (TEIXEIRA et al., 1987).
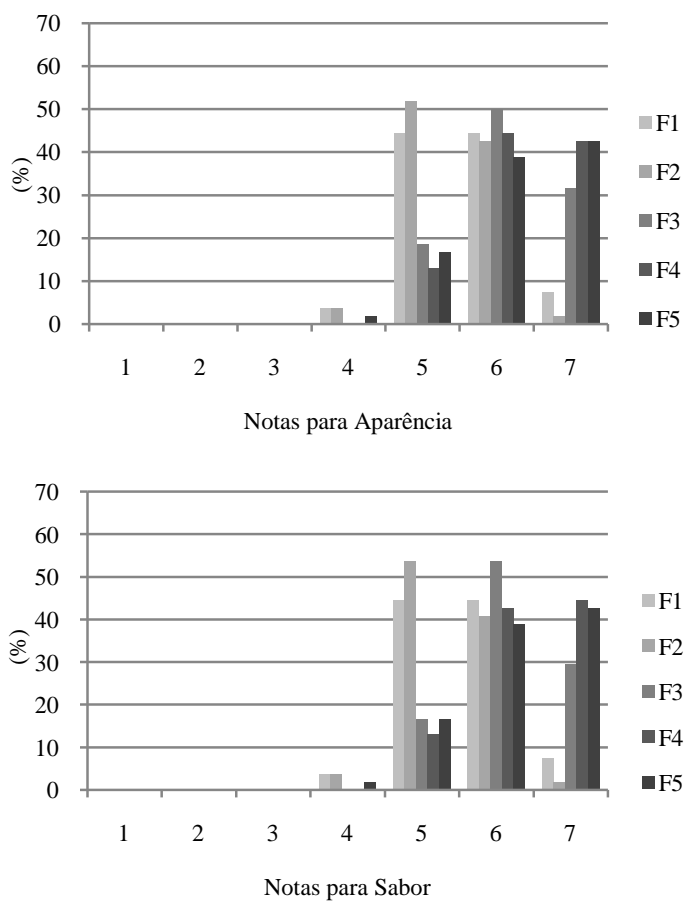

Resultados similares foram constatados por Devereux et al. (2003), avaliando a aceitação de pães adicionados inulina (20 a 80\%), entre adultos. Entretanto, as avaliações de aceitação global (F1, F2 e F3) e intenção de compra (F1) mostram índices menores.

A alta aceitabilidade dos pretzels com adição de inulina demonstra a possibilidade da inclusão de fibras em alimentos para o consumo de crianças em fase escolar. Efeito muito positivo, já que a ingestão adequada de fibras alimentares reduz a glicemia e o risco de doenças cardiovasculares. Além disso, melhora a constipação intestinal, promove a saciedade (SAAD, 2006) e absorção de cálcio ósseo (GRIFFIN et al., 2003).
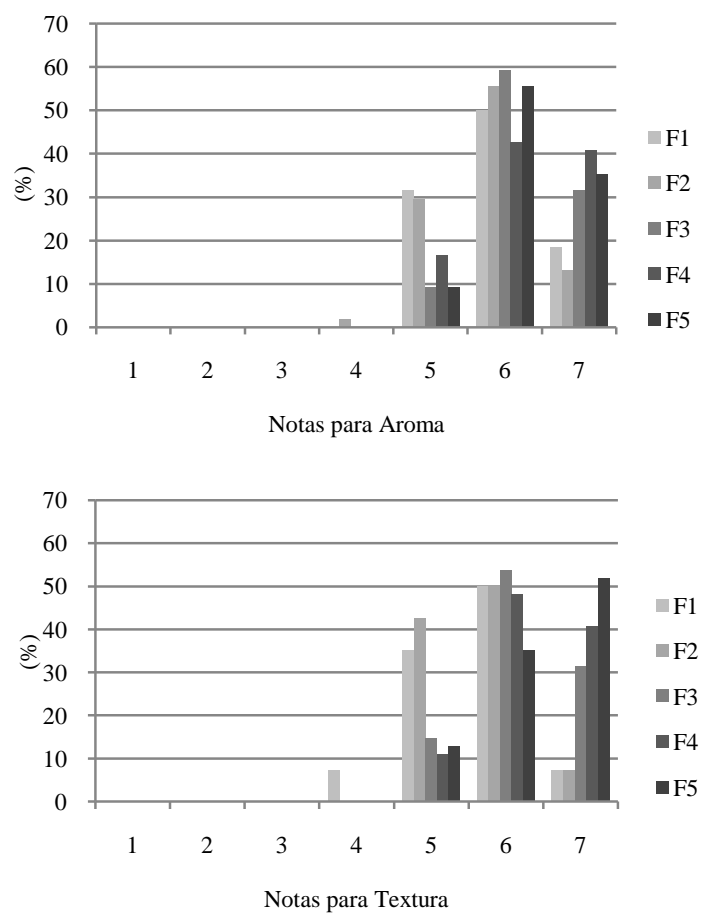

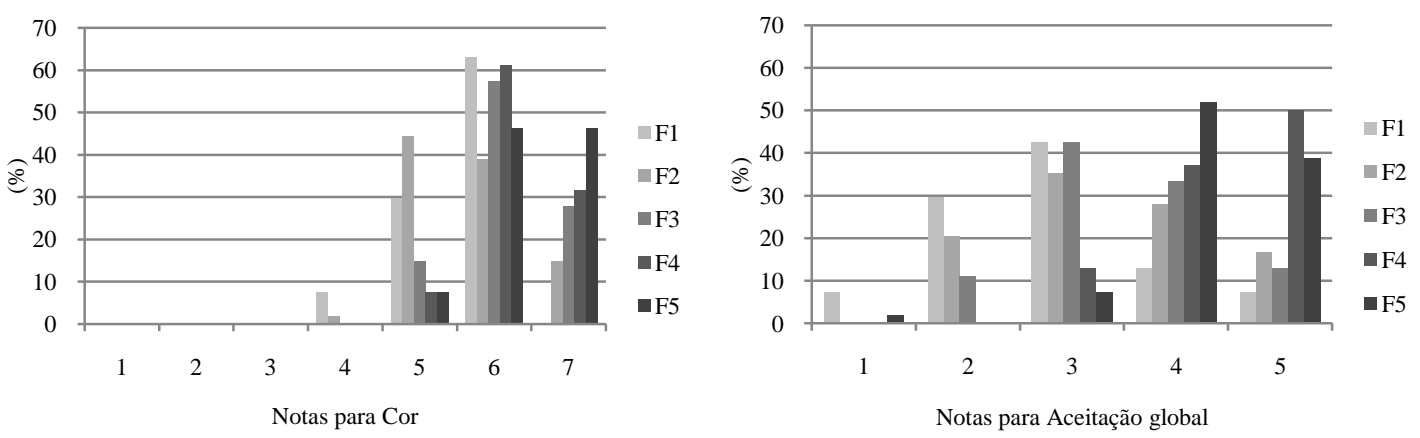

Figura 1 - Distribuição dos provadores pelos valores hedônicos obtidos na avaliação dos atributos aparência, aroma, sabor, textura, cor e aceitação global das formulações de pretzel integral padrão - 0\% (F1) e adicionadas de 1\% (F2), 2\% (F3), 3\% (F4) e $4 \%$ (F5) de inulina.

A maioria das porcentagens das notas conferidas pelos provadores concentraram-se em 6 ("muito bom"), o que demonstra que as formulações foram, em geral, bem aceitas pelos provadores. Entretanto, destaca-se que as amostras com menores teores de inulina (F1 e F2) apresentaram elevadas porcentagens de julgamentos na nota 5 ("bom"), o que evidencia uma aceitação inferior. Para a avaliação de aceitação global, as notas se concentraram acima de 4 ("gostei"), com destaque para F4 e F5.

Conforme Dutcosky (2011), a adição de novos ingredientes em um produto pode melhorar sua qualidade, além de aumentar o valor nutritivo, colaborando para aprimorar atributos sensoriais como cor, aparência, aroma, sabor, textura, consistência e a interação dos diferentes componentes, fato que aumenta a aceitabilidade e possibilidade de compra do produto. Estes efeitos foram confirmados na presente pesquisa. Destaca-se também, que um consumo adequado de prebióticos na infância pode auxiliar para o controle e estabilização da microbiota intestinal, resistência gastrintestinal a colonização por patógenos, digestão da lactose, estimulação do sistema imune e redução da constipação intestinal, dentre outros benefícios (SAAD, 2006).

Por meio da Figura 2 verificam-se os resultados do teste de comparação múltipla das amostras, comparadas com um produto similar vendido comercialmente. 


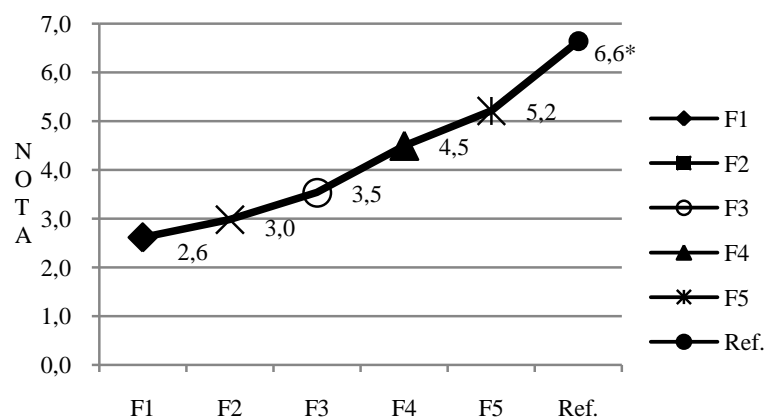

*diferença significativa pelo teste de médias de Dunnett $(\mathrm{p}<0,01)$.

Figura 2 - Notas médias do teste de comparação múltipla das amostras de pretzel integral padrão - 0\% (F1) e adicionadas de $1 \%$ (F2), $2 \%$ (F3), 3\% (F4) e 4\% (F5) de inulina, comparadas com um produto vendido comercialmente (referência), em relação ao sabor.

Todas as formulações foram consideradas com sabor inferior $(\mathrm{p}<0,05)$ à marca comercializada. Segundo Kaur e Gupta (2002), isso ocorre, principalmente, devido ao menor teor adoçante da inulina (30 a 50\%) quando comparada ao açúcar. O fato das formulações adicionadas de inulina se apresentarem com sabor mais próximo ao produto referência pode ser explicado, pois houve uma melhora considerável na textura (aumento da maciez) após a adição de inulina, o que promoveu uma consistência mais próxima ao produto comercial. Resultados similares foram observados por Morris e Morris (2012), onde a adição da inulina (6\%) em pães melhorou a elasticidade e a resistência à deformação.
Uma das questões fundamentais para a área de alimentos é o desenvolvimento de um produto de boa qualidade e que apresente elevada aceitabilidade pelos consumidores. Em razão disso, a amostra F5 (4\% de inulina) foi selecionada para fins de comparação, juntamente com a padrão (F1), por ser aquela com o maior teor de inulina e com aceitação superior a padrão em todos as características.

\section{Composição físico-química}

Na Tabela 3, observam-se os valores da composição físico-química do pretzel padrão e com adição de $4 \%$ de inulina. 
Tabela 3 - Composição físico-química e valores diários recomendados - VD* (porção média de 50 g) de pretzel integral padrão (F1) e com adição de $4 \%$ de inulina (F5)

\begin{tabular}{|c|c|c|c|c|}
\hline \multirow[b]{2}{*}{ Avaliacão } & \multicolumn{2}{|c|}{ F1 } & \multicolumn{2}{|c|}{ F5 } \\
\hline & Média $\pm D P$ & $\mathrm{VD}(\%)^{*}$ & Média $\pm D P$ & $\operatorname{VD}(\%)^{*}$ \\
\hline Umidade (\%) & $14,26 \pm 0,02^{b}$ & ND & $25,98 \pm 0,01^{\mathrm{a}}$ & ND \\
\hline Cinzas $\left(\mathrm{g} .100 \mathrm{~g}^{-1}\right)^{* *}$ & $1,48 \pm 0,02^{\mathrm{a}}$ & ND & $1,40 \pm 0,01^{\mathrm{a}}$ & ND \\
\hline Proteínas $\left(\mathrm{g} .100 \mathrm{~g}^{-1}\right) * *$ & $13,97 \pm 0,09^{\mathrm{a}}$ & 8,90 & $13,45 \pm 0,02^{\mathrm{a}}$ & 8,56 \\
\hline Lipídios $\left(\mathrm{g} .100 \mathrm{~g}^{-1}\right)^{* *}$ & $3,75 \pm 0,04^{\mathrm{a}}$ & 3,33 & $3,72 \pm 0,02^{\mathrm{a}}$ & 3,45 \\
\hline Carboidratos (g. $\left.100 \mathrm{~g}^{-1}\right) * *$ & $66,54 \pm 0,15^{\mathrm{a}}$ & 10,60 & $55,45 \pm 0,23^{b}$ & 8,77 \\
\hline Calorias (kcal.100g $\left.\mathrm{g}^{-1}\right)^{* *}$ & $357,97 \pm 0,89^{\mathrm{a}}$ & 8,60 & $301,13 \pm 0,98^{b}$ & 7,23 \\
\hline Fibra alimentar $\left(\mathrm{g} .100 \mathrm{~g}^{-1}\right) * * *$ & 2,12 & 7,03 & 6,00 & 19,88 \\
\hline
\end{tabular}

Letras diferentes na linha indicam diferença significativa pelo teste de t de student ( $\mathrm{p}<0,05)$; *VD: nutrientes avaliados pela média da DRI (2005), com base numa dieta de 2.080,93 kcal/ dia; **Valores calculados em base seca; ***Cálculo teórico em base úmida (TACO, 2011; BENEO ${ }^{\circledR}$, 2015); DP: desvio padrão da média; ND: não disponível.

Maiores teores $(\mathrm{p}<0,05)$ de umidade foram verificados na formulação com adição de inulina (F5). Esse fato ocorreu, pois este ingrediente apresenta elevada higroscopicidade, podendo absorver acima de 20 vezes seu peso em água (THEBAUDIN et al., 1997). Entretanto, ambas as formulações estão de acordo com a Resolução - CNNPA n ${ }^{\circ} 12$, de 1978, que estabelece o máximo de 30\% de umidade em pães (BRASIL, 1978). Resultados que corroboram com Silva et al. (2010), que avaliaram as características de pães com adição de frutooligossacarídeos $(4,5 \%)$.

Não houve diferença significativa entre os conteúdos de cinzas, proteínas e lipídios em ambas as amostras ( $>>0,05)$, pois o açúcar e a inulina apresentam apenas traços desses nutrientes em sua composição (TACO, 2011; BENEO $\left.^{\circledR}, 2015\right)$. Estes dados concordam com Wang et al. (2002), que adicionaram frutooligossacarídeos (até 3\%) em pães.
Maiores quantidades de carboidratos e calorias $(p<0,05)$ foram verificadas em F1. Esse resultado pode ser explicado, pois a inulina apresenta menores teores desses nutrientes em sua composição (97 g.100g ${ }^{-1}$ de CHO e 1,5 $\mathrm{kcal} / \mathrm{g}$, respectivamente) $\left(\right.$ BENEO $\left.^{\circledR}, 2015\right)$, quando comparada ao açúcar $\left(99,5 \mathrm{~g} \cdot 100 \mathrm{~g}^{-1} \mathrm{de}\right.$ $\mathrm{CHO}$ e $3,87 \mathrm{kcal} / \mathrm{g}$, respectivamente) (TACO, 2011). Destaca-se que o pretzel adicionado de inulina é composto por um maior teor de carboidratos complexos, os quais apresentam maiores benefícios à saúde (LERMAN et al., 1994).

Em relação às fibras, cabe enfatizar que o teor verificado na formulação F5 $(6,00$ g. $\left.100 \mathrm{~g}^{-1}\right)$, expressa um aumento de $183 \%$ em relação a F1. Isso se deve, principalmente, ao alto teor de fibras (97\%) presente na inulina $\left(\right.$ BENEO $^{\circledR}$, 2015). Esse resultado torna o produto uma nova alternativa alimentar para a faixa etária em questão, visto que as crianças 
apresentam um baixo consumo de frutas e verduras in natura (COSTA et al., 2012).

De acordo com a Legislação Brasileira (BRASIL, 2012), um produto é considerado como fonte de fibra alimentar quando apresenta em sua composição química no mínimo 3\% de fibras. Já, quando o conteúdo for de $6 \%$ o alimento pode ser classificado com alto teor em fibras. Assim, pode-se considerar F5 como um produto com alto teor de fibra alimentar.

\section{CONCLUSÕES}

A elaboração dos produtos permitiu comprovar que um nível de adição de até $4 \%$ de inulina em pretzels integrais (redução de 100\% do açúcar) foi bem aceito pelos provadores infantis, obtendo-se aceitação sensorial semelhante ao produto padrão.

A adição de $4 \%$ de inulina em pretzels integrais proporcionou redução nos teores de carboidratos e calorias e aumento de umidade. Foi possível, também, elevar o aporte de fibras, melhorando o perfil nutricional do produto. Assim sendo, a inulina pode ser considerada um potencial ingrediente com propriedades funcionais para adição de pães e similares, podendo ser oferecidas aos consumidores infantis com altas expectativas de comercialização.

\section{AGRADECIMENTOS}

Os autores agradecem à Fundação Araucária de Apoio ao Desenvolvimento Científico e Tecnológico do Estado do Paraná, pela bolsa concedida (Programa Institucional de Apoio a Inclusão Social - Pesquisa e Extensão Universitária).

\section{REFERÊNCIAS}

AOAC International. Official Methods of Analysis of AOAC International. 18 ed. 4 rev. Gaithersburg: AOAC, 2011. 1505p.

AQUINO, J.D.S.; PONTES PESSOA, D.C.N.; OLIVEIRA, C.E.V.; STAMFORD, T.L.M.

Processamento de biscoitos adicionados de óleo de buriti (Mauritia flexuosa L.): uma alternativa para o consumo de alimentos fontes de vitamina A na merenda escolar. Revista de Nutrição, v.25, n.6, p.765-774, 2012.

\section{BENEO $^{\circledR}$ HP. Product Sheet Beneo ${ }^{\circledR}$ HP,} Orafti, DOC.A4-05*01/02-B. Disponível em: http://www.orafti.com. Acesso em: 14/06/2015.

BRASIL, J.A.; SILVEIRA, K.C.; SALGADO, S.M.; LIVERA, A.V.S.; FARO, Z.P.;

GUERRA, N.B. Effect of the addition of inulin on the nutritional, physical and sensory parameters of bread. Revista Brasileira de Ciências Farmacêuticas, v.47, n.1, p.185-191, 2011.

BRASIL, Ministério da Saúde. Agência Nacional de Vigilância Sanitária (ANVISA). Resolução - CNNPA no 12, de 1978. Normas técnicas especiais relativas a alimentos (e bebidas). Diário Oficial da União; Poder Executivo, Brasília, DF, 1978. 
BRASIL. Ministério da Saúde. Agência Nacional de Vigilância Sanitária (ANVISA). Métodos físico-químicos para análise de alimentos. 4 ed. Brasília: Ministério da Saúde, 2005. 1018p.

BRASIL. Ministério da Saúde. Agência Nacional de Vigilância Sanitária (ANVISA).

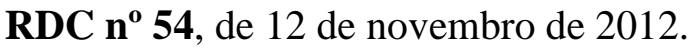
Regulamento Técnico sobre Informação Nutricional. Diário Oficial da União; Brasília, DF, 2012.

CAZARIN, C.B.B.; SILVA, J.K.; COLOMEU, T.C.; ZOLLNER, R.L.; JUNIOR, M.R.M. Capacidade antioxidante e composição química da casca de maracujá (Passiflora edulis). Ciência Rural, v.44, n.9, p.1699-1704, 2014.

CENCIC, A.; CHINGWARU, W. The role of functional foods, nutraceuticals, and food supplements in intestinal health. Revista de Nutrição, v.2, n.6, p.611-625, 2010.

COSTA, A.; BARAUNA, A.C.; BERTIN, R.L.; TAVARES, L.B.B. Flaxseed flour addition on fatty acid profile and sensory properties of brazilian cheese roll. Ciência e Agrotecnologia, v.36, n.4, p.431-438, 2012.

CUNHA, A.O. Cadeia produtiva do pão : fontes informacionais utilizadas no planejamento de novos produtos. Porto Alegre 2012. Disponível em: https://www.lume.ufrgs.br/bitstream/handle/10 $183 / 54255 / 000855773$.pdf? sequence $=1$. Acesso em: 01/05/2015.

DALONSO, N.; IGNOWSKI, E.; MONTEIRO, C.M.A.; GELSEICHTER, M.; WAGNER, T.M.; SILVEIRA, M.L.L.; SILVA, A.K.S. Extração e caracterização de carboidratos presentes no alho (Allium sativum L.): proposta de metodologia alternativa. Food Science and Technology, v.29, n.4, p.793-797, 2009.

DEVEREUX, H.M.; JONES, G.P.; MCCORMACK, L.; HUNTER, W.C.
Consumer acceptability of low fat foods containing inulin and oligofructose. Journal of Food Science, v.68, n.5, p.1850-1854, 2003.

DIETARY REFERENCE INTAKE (DRI). Dietary Reference Intake for Energy, Carbohydrate, Fiber, Fat, Fatty Acids, Cholesterol, Protein, and Amino Acids (Macronutrients). Washington, DC: National Academy Press; 2002, 936p.

DIETARY REFERENCE INTAKES (DRI). Dietary Reference Intakes for energy, carbohydrate, fiber, fat, fatty acids, cholesterol, protein and amino acids. Washington: National Academy Press, 2005. 1331p.

DUTCOSKY, S.D. Análise sensorial de alimentos. 3. ed. Curitiba: Champagnat, 2011. $427 p$.

ELLEUCH, M.; BEDIGIAN, D.; ROISEUX, O.; BESBES, S.; BLECKER, C.; ATTIA, H. Dietary fibre and fibre-rich by-products of food processing: Characterization, technological functionality and commercial applications: A review. Food Chemistry, v.124, n.2, p.411421, 2011

FLAMM, G.; GLINSMANN, W.; KRITCHEVSKY, D.; PROSKY, L.;

ROBERFROID, M. Inulin and Oligofructose as dietary fiber: a review of the evidence. Critical Reviews in Food Science and Nutrition, v.41, n.5, p.353-362, 2001.

GANORKA, P.M.; JAIN, R.K. Flaxseed - a nutritional punch. International Food

Research Journal, v.20, n.2, p.519-525, 2013.

GOMES, J.C.; OLIVEIRA, G.O. Análises físico-químicas de alimentos. Viçosa: UFV, 2011. 303p.

GRIFFIN, I.J.; HICKS, P.M.D.; HEANEY, R.P.; ABRAMS, S.A. Enriched chicory inulin increases calcium absorption mainly in girls 
with lower calcium absorption. Nutrition Research, v.23, n.7, p.901-909, 2003.

IRANSHAHIL, M.; ARDEBILI, S.M.S.; ARDAKANI, S.Y. Effect of inulin and $\beta$ glucan on the physicochemical, rheological, and sensory properties barbari bread. Monireh Iranshahi et al Int. Journal of Engineering Research and Applications, v.4, n.6, p.90-97, 2014.

KAUR, N.; GUPTA, A.K. Applications of inulin and oligofructose in health and nutrition. Journal of Biosciences, v.27, n.7, p.703-714, 2002.

KIP, P.; MEYER, D.; JELLEMA, R.H. Inulins improve sensoric and textural 14 properties of low-fat yoghurts. International Dairy Journal, v.16, n.9, p.1098-1103, 2006.

LERMAN, I.G.; IDHAZO-CERRO,S.; ZAMORA-GONZALEZ, J.; CARDOSOSALDAÑA, G.; POSADAS-ROMERO, C. Effect of a higt monounsaturated fat diet enriched with avocado in NIDDM patients. Diabetes Care, v.17, n.4, p.311-315, 1994.

LIPILINA, E.; GANJI, V. Inconporation of ground flaxseed into bakery products and effect on sensory and nutritional characteristics - a pilot study. Journal of Food Service, v.20, n.1, p.52-59, 2009.

MANDALA, I.; POLAKI, A.; YANNIOTIS, S. Influence of frozen storage on bread enriched with different ingredients. Journal of Food Engineering, v.92, n.2, p.137-145, 2009

MASSODI, L.; KHALID BASHIR, V.A. Fortification of Biscuit with Flaxseed: Biscuit Production and Quality Evaluation. Journal of Environmental Science, v.1, n.5, p.6-9, 2012.

MELLO, C.S.; FREITAS, K.C.; TAHAN, S.; BATISTA-MORAIS, M. Consumo de fibra alimentar por crianças e adolescentes com constipação crônica: influência da mãe ou cuidadora e relação com excesso de peso. Revista Paulista de Pediatria, v.28, n.2, p.188-193, 2010.

MERRILL, A.L.; WATT, B.K. Energy values of foods: basis and derivation. Agricultural Handbook, n.74, Washington: USDA, 1973. 106p.

MINIM, V.P.R. Análise Sensorial: estudo com consumidores. 2 ed. Viçosa: UFV, 2010. 308 p.

MONTEIRO, C.L.B. Técnicas de avaliação sensorial. 2d. Curitiba: CEPPA-UFPR, 1984. $101 \mathrm{p}$.

MORAES, E.A.; DANTAS, M.I.S.; MORAIS, D.C.; SILVA, C.O.; CASTRO, F.A.F.; MARTINO, H,S,D,M.; RIBEIRO, S.M.R. Sensory evaluation and nutritional value of cakes prepared with whole flaxseed flour. Ciência e Tecnologia de Alimentos, v.30, n.4, p.974-979, 2010 .

MORRIS, C.; MORRIS, G.A. The effect of inulin and fructo-oligosaccharide supplementation on the textural, rheological and sensory properties of bread and their role in weight management: A review. Food Chemistry, v.133, n.2, p.237-248, 2012.

OLIVEIRA, N.M.A.L.; MACIEL, J.F.; LIMA, A.S.; SALVINO, E.M.; MACIEL, C.E.P.; NERY-OLIVEIRA, D.P.M.; FARIAS, L.R.G. Características físico-químicas e sensoriais de pão de forma enriquecido com concentrado proteico de soro de leite e carbonato de cálcio. Revista Instituto Adolfo Lutz, v.70, n.1, p.16-22, 2011 .

PAULA, N.S.; NATAL, D.I.G.; FERREIRA, H.A.; DANTAS, M.I.S.; RIBEIRO, S.M.R.; MARTINO, H.S.D. Characterization of cereal bars enriched with dietary fiber and omega 3. Revista Chilena de Nutrición, v.40, n.3, p.269-273, 2013. 
PINTO, A.L.D.; PAIVA, C.L.

Desenvolvimento de uma massa funcional pronta para tortas utilizando o método de Desdobramento da Função Qualidade (QFD).

Ciências e Tecnologia de Alimentos, v.30, n.1, p.36-43, 2010.

PONTES AIRES, A.P.; LOPES-SOUZA, C.C.; BENEDETTI, F.J.; BLASI, T.C.; KIRSTEN, V.R. Consumption of processed foods in preschool children. Revista da AMRIGS, v.55, n.4, p.50-355, 2011.

RESURRECCION, A.V.A. Consumer Sensory Testing for Product Development. Gaithersburg: Aspen Publishers, 1998. 276p.

RUBILAR, M.; GUTIERREZ, M. VERDUGO, C.; SHENE, J.S. Flaxseed as a source of functional ingredients. Journal of Soil Science and Plant Nutrition, v.10, n.3, p.373-377, 2010.

SAAD, N.; DELATTRE, C.; URDACI, M.; SCHMITTER, J. M.; BRESSOLLIER, P. An overview of the last advances in probiotic and prebiotic field. Food Science and Technology, v.50, n.1, p.1-16, 2013.

SAAD, S.M.L. Probióticos e prebióticos: o estado da arte. Revista Brasileira de Ciências Farmacêuticas, v.42, n.1, p.1-16, 2006.

SILVA, F.A.S.; DUARTE, M.E.M.; CAVALCANTI-MATA, M.E.R.M. Nova metodologia para interpretação de dados de análise sensorial de alimentos. Engenharia Agrícola, v.30, n.5, p.967-973. 2010

SILVA, L.M.R.; LIMA, A.S.; MAIA, G.A.; RODRIGUES, M.C.P.; FIGUEIREDO, R.W.; SOUZA, P.H.M. Archivos Latinoamericanos de Nutrición, v.61, n.2, p.209-215, 2011.
Tecnologia de Alimentos, v.32, n.2, p.374380, 2012.

SOUZA-BORGES, P.K.; SOKEI, F.R.; SPAGNOLL, T.D.; CONTI-SILVA, A.C.

Características químicas, físicas e sensoriais de bolos de laranja e pães adicionados de inulina e oligofrutose. Semina: Ciências Agrárias, v.34, n.6, p.2837-2846, 2013.

TABELA BRASILEIRA DE COMPOSIÇÃO DE ALIMENTOS (TACO). Tabela brasileira de composição de alimentos. 4. ed. Campinas: NEPA-UNICAMP, 2011. 161p.

TEIXEIRA, E.; MEINERT, E.; BARBETTA, P.A. Análise sensorial dos Alimentos.

Florianópolis: UFSC, 1987. 182p.

THEBAUDIN, J.Y.; LEFEBVRE, A.C.;

HARRINGTON, M.; BOURGEOIS, C.M.

Dietary fibres: nutritional and technological interest. Trends in Food Science \&

Technology, v.8, n.2, p.41-48, 1997.

WANG, J.; ROSSEL, C.M.; BARBER, C.B. Effect of the addition of different fibres on dough performance and bread quality. Food Chemistry, v.79, n.2, p.221-226, 2002.

SIMBALISTA, R.L.; FROTA, K.M.G.;

SOARES, R.A.M.; AREAAS, J.A.G. Effect of storage and processing of Brazilian flaxseed on lipid and lignan contents. Ciência e 